\title{
Design of Oscilloscope Monitoring Program
}

\author{
Yao Zhang ${ }^{1, a}$ and Yuanshao Hou ${ }^{1, b}$ \\ ${ }^{1}$ College of Information Engineering, Zhengzhou University Of Industrial Technology, Xinzheng, \\ China \\ a304963405@qq.com, b516063795@qq.com
}

Keywords: Keyboard; Coding switch; Analog-digital conversion; Monitor

\begin{abstract}
High-powered C8051F020 MCU is used as controller chip. Forty oscilloscope buttons, three coding switches and four potentiometer states on oscilloscope panel are monitored. The working principle, the hardware circuit connecting to MCU and the implementation of software programming of keyboard, coding switches and potentiometer are introduced. The buttons are used by programming method that is one key ambiguity. The flow chart of key code matching subroutine is also given. In $\mathrm{AD}$ conversion section, the method of removing the four lower to filter is proposed, it is reliable and feasible in occasion of less precision through experiment.
\end{abstract}

\section{Introduction}

Monitoring program is responsible for the work that allocating and scheduling resources of all hardware and software in system. The monitoring program provides customer interface, which make users obtain friendly work environment, and it is an important part of system designing.

\section{The Keyboard Working Principle of One Key Ambiguity}

In one key ambiguity, a command is not composed of a button but a sequence of keys. That is to say, the interpretation of a key, is not only depends on the current key, but also depends on the previous one. Therefore, the monitoring program of one key ambiguity judges a key sequence (not a key) whether constitutes a lawful order. If there is a lawful order, executing command, or waiting for the new key input. Therefore, the keyboard management program of one key ambiguity mainly to solve two issues that recognizing sequence of keyboard keys and finding the corresponding procedures according to the keyboard key sequences [1].

The above problems can be solved by "One Figure Three table". Namely, establishing a bond graph, it is to perform by relying on the analysis program status table, program inlet scale and action case subroutines table [2].

\section{The Working Principle of Encode Switch}

Encode switch has three pins or five pins. Two of them have press function, the other three have the left lateral and right lateral function to control encode switch. Two different length static plates are connected with pin 1 and pin 2. A dynamic plate with 12 or 24 gears is connected with pin 3 . It will occur four states when the encode switch is rotary. Namely, pin 3 is connected to pin 1 . Pin 3 , pin 2 and pin 1 are connected to each other, pin 3 is connected to pin 2 . Pin 3, pin 2 and pin 1 are disconnected from each other. Fig. 1 shows code switch outline drawing.

In practice, pin 3 grounding is data input. Pin 1 and pin 2 which are data output are connected with I/O of MCU. In this scheme, there are three code switches. One of them make the connection between pin 1 and P4.0 of MCU. Pin 2 is connected with P4.1 of MCU. When code switch is left lateral and right lateral, P4.0 and P4.1 will periodically generated waveform shown in Fig. 2. If the 12-point pulse potentiometer rotates one circle, it will produce 12 groups of the waveform. The 24-point pulse potentiometer will produce 24 groups of the waveform. A set of waveforms (or a cycle) contains four work status. Therefore, as long as detecting the waveform of P4.0 and P4.1, It can identify whether the pulse potentiometer rotation, left-handed or right-handed [3]. 


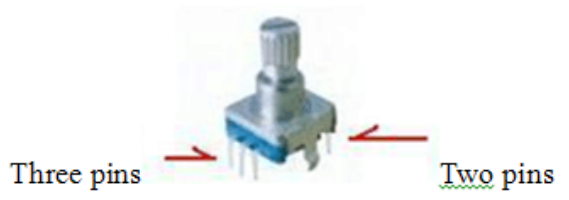

Figure 1. Code switch outline drawing
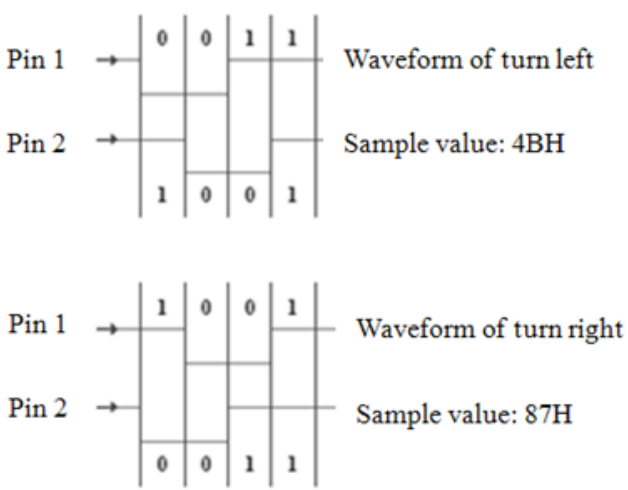

Figure 2. Work waveform graph of code switch

\section{The Introduction of C8051F020 ADC0}

It is usually in tracking mode before starting ADC. If the bit 6 of control register ADCOCN is " 0 ", it is always in the tracking mode (The four kinds of start-up mode can be faster than flying start three system clock at this time); If it is "1", there are four kinds of tracking start-up mode to be chosen, that assigned the bit 3 2 of ADC0CN: 00 is track when writing to ADBUSY 1 (software command); 01 is overflow tracking of timer 3; 10 is rising track of CNVSTR (external signal); 00 is overflow tracking of timer 2 [4].

\section{Design of System Hardware}

$6 * 6$ matrix keyboard is used in keyboard part, P7.0-P7.5 is row line, P3.0-P3.5 is column line. The intersection of $\mathrm{P} 3.0$ and $\mathrm{P} 7.0$ is a key, $\mathrm{P} 7$ port meets $10 \mathrm{~K}$ pull-up resistor to $3.3 \mathrm{~V}$. Feet 1,2 of three coding switches connected to I/O pin of MCU, choosing P4.0-P4.5, the feet 3 grounded, 4, 5 pin are used as the keys [5]. Only meeting P4.0 and P4.1 pin code switch acts an example, the circuit diagram is shown in Fig. 3. Analog and digital conversion section uses an internal voltage reference, it will be connected to the VREF pin can VREF0 pin. Using potentiometer adjusts analog input, single-ended input, potentiometer resistance is $10 \mathrm{~K}$, a typical value of the reference voltage is $2.43 \mathrm{~V}$, and the power supply voltage is $3.3 \mathrm{~V}$. In order to achieve the maximum reference voltage, it needs that a resistor which resistance value is approximately $3.58 \mathrm{~K}$ connected to the potentiometer in series, and then connected to the analog port. The hardware circuit is shown in Fig. 4, the potentiometer 4, 5 feet are used as keys.

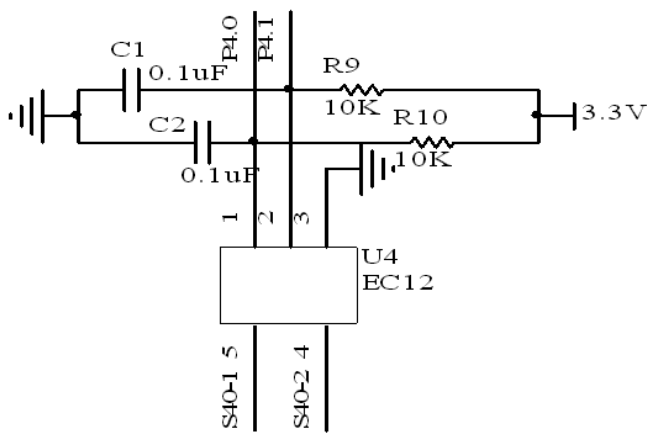

Figure 3. Interface circuit of coding switch and C8051F020 MCU 


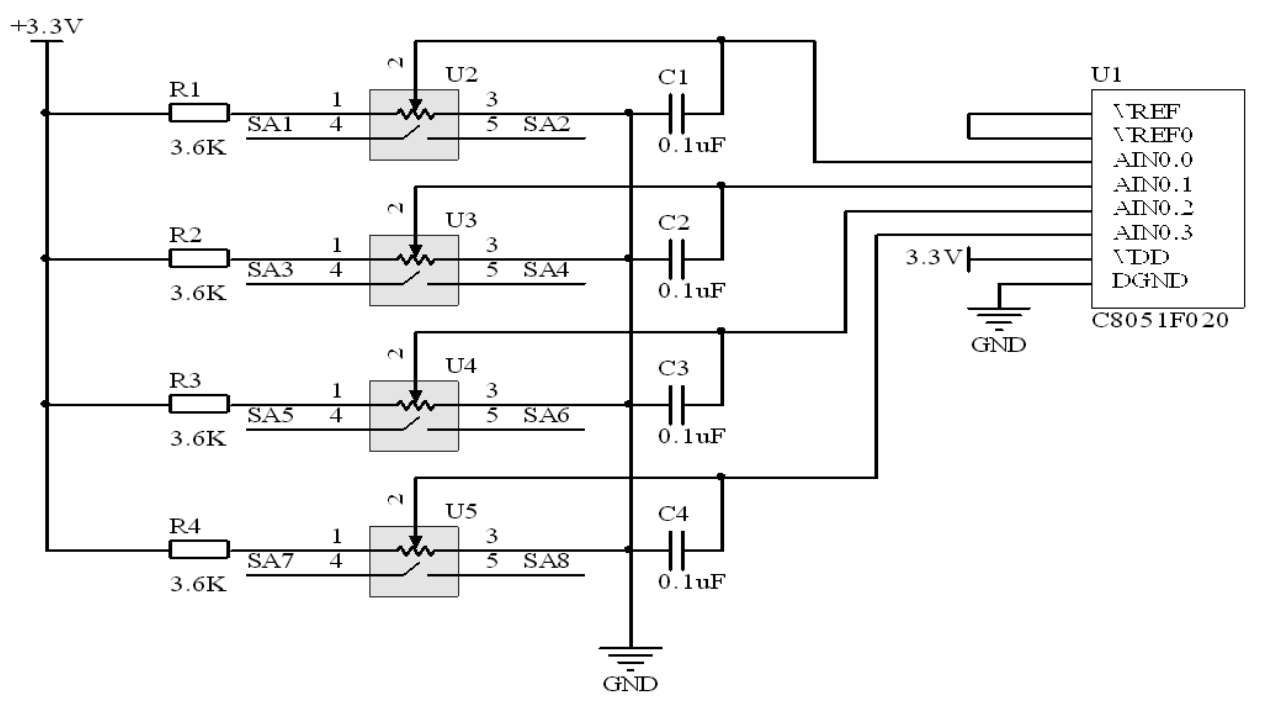

Figure 4. Interface circuit of potentiometer and C8051F020 MCU

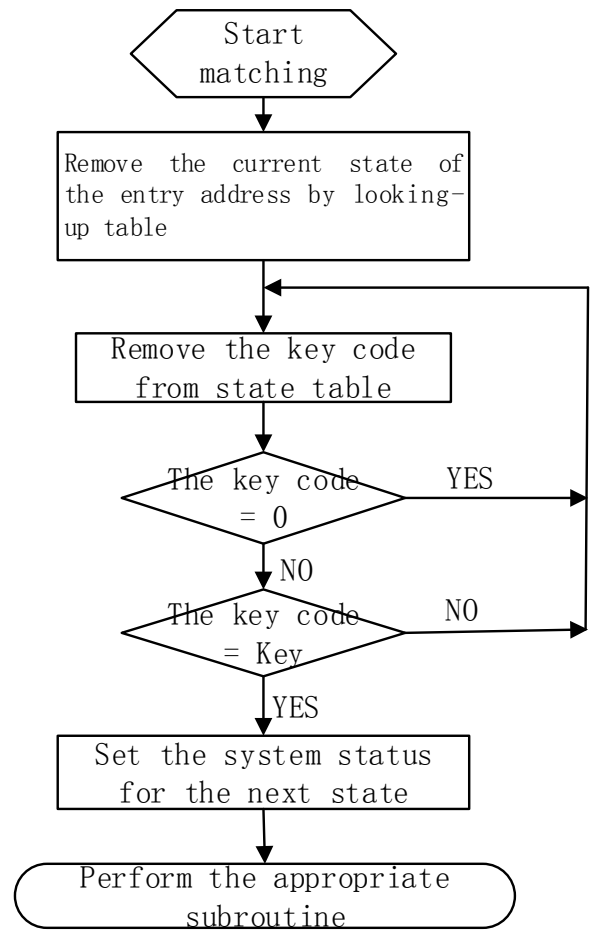

Figure 5. Flow chart of key code matching subroutine

\section{Software Design}

The Keyboard Programming of One Key Ambiguity. In analysis of keyboard, the current state of the keyboard is saved by a status register, the address of analysis program state table is found in the analysis program entry table according to the current state of the value. It will access to analysis program state table from the address to find matching values. The next state is taken to cell of the current, taking the action number, action subroutine entry address is calculated according to action number, and then perform the appropriate subroutine [6-8]. A flow chart of key-code matching subroutine is shown in Fig. 5.

Program Design of Coding Switching. We can see from Fig. 2, pins 1 and 2 are both in the high level sometimes. If pin 1 gets high level after pin 2, it is left-handed. If pin 2 gets high level after pin 1, it is right-handed. The state of pin 1 and pin 2 is judged according to this feature when programming. Pin 1 meet $\mathrm{P} 4.0$ and pin 2 meet $\mathrm{P} 4.1$ make an example. 
int CodingsWitchPolling1()

\{ static uchar Aold,Bold; // Two variables are defined to save two pin level of coding switch get from first call this method.

static uchar st1; $\quad / / \quad$ One variable is defined to save that If there were two pins are both high state before

CodingsWitch_A=P4\&0x02;

CodingsWitch_B=P4\&0x01;

If (CodingsWitch_A\&\&CodingsWitch_B)

st $1=1$;

if (st1) // Perform the following steps if the st is 1

\{ if(CodingsWitch_A==0\&\&CodingsWitch_B==0) // Perform the following steps if the two pins of current coding switch are both low level

\{ if(Bold) // It is high that means coding switch turns to increasing direction

$\{\mathrm{st} 1=0 ; \mathrm{tmp} 1++; \quad\}$

if(Aold) // It is high that means coding switch turns to decreasing direction

$\{\quad$ st $1=0 ;$ tmp1--; $\}\}$ \}

Aold $=$ CodingsWitch_A; Bold $=$ CodingsWitch_B;

return tmp $1 ; \quad\}$

ADC Software Design. As the device workload is not large, the query is used in software design. MCU constantly query the state of keyboard, coding switches and potentiometers, if there is a change, the device will transmit operation information to the main ARM MCU, pending the main MCU to deal with. Since the analog to digital conversion speed of MCU is very fast, the delay is added in the program so that the change of analog to digital conversion can be observed. As it is not consider filtering in hardware design, the filtering is achieved by software. General filtering method is limiter filtering, median filtering, arithmetic mean filtering method, and now a new filtering method is proposed. Since 12 bit AD is used, only 8 bit AD can achieve the accuracy, the method that get rid of the low 4 bits is used to achieve the filtering. Due to limited space, only part of the program is shown, taking AIN0 as an example:

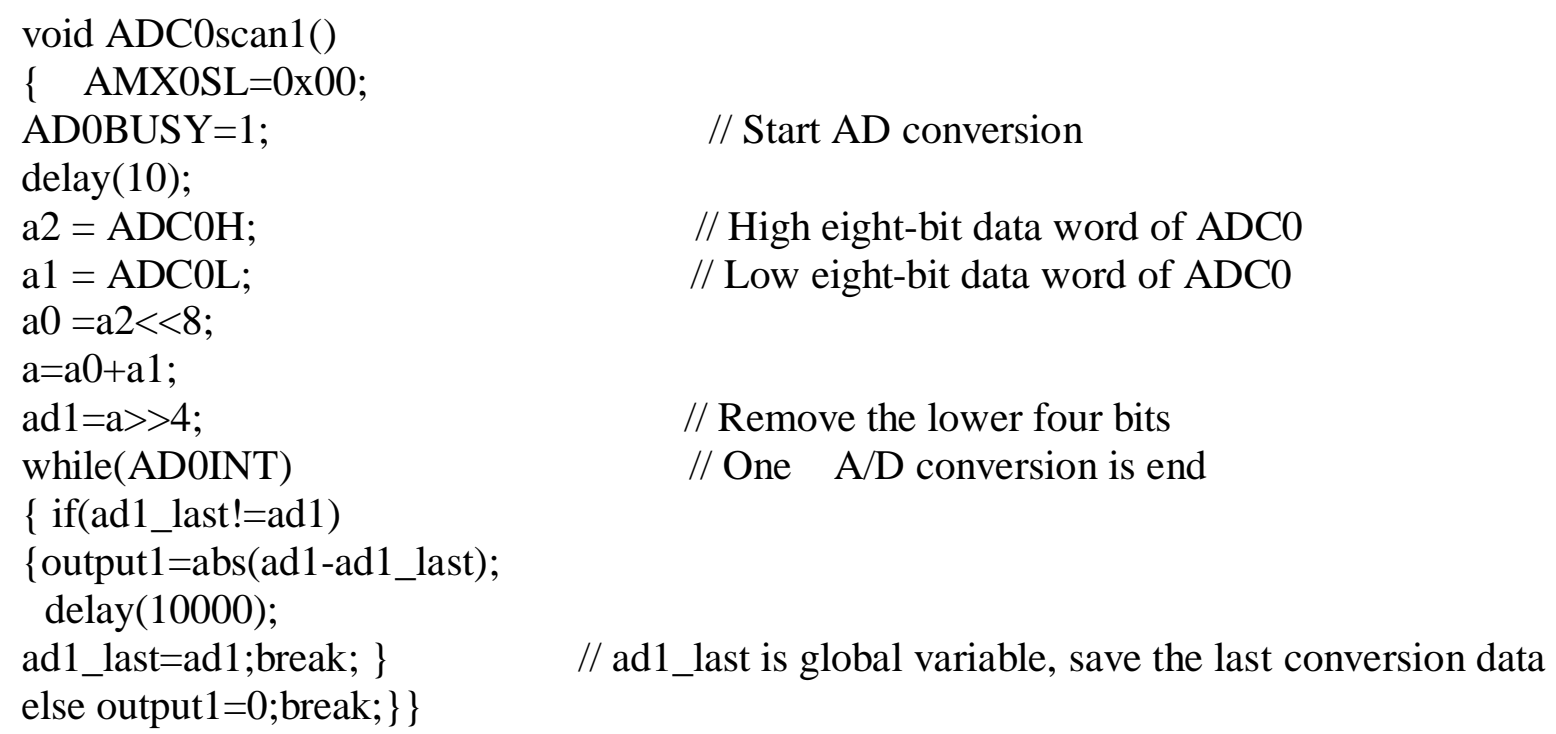

\section{Conclusion}

The key management program of a multi-key described in this article can be used in multi-button smart meter. The programming method of coding switch is simple and understandable [9]. In AD conversion section, software filtering method that removing the lower 4 bits is reliable and feasible, and is ideal for less precision occasion [10]. These three parts form a complete monitoring program, 
when MCU monitors changes of a section, it will pass its operation information to the main ARM CPU for the main CPU to deal with.

\section{References}

[1] W.J. Yuan, K.X. Zhao and L.W. Liu. Wireless remote car alarm based on GPRS [J]. Journal of Changchun University of Technology, Vol.29 (2008) No.4, p.391.

[2] S.C. Sun, H.F. Han. Design of the microcomputer monitoring program for the monitoring and controlling system. Microcontrollers \& Embedded Systems, (2008) No.1, p.66.

[3] P.A. Wang. Application of rotary coding switch in MCU [J]. Journal of Yangtze University (Natural Science Edition), Vol.10 (2013) No.13, p.88.

[4] G.M. Han. The Development of the Machine Tool NC System Based on C8051F020 MCU [J]. Electronics World, (2011) No.11, p.25.

[5] W.C. Pan. Software and Hardware Design Based on Menu Interface in Intelligent Instruments. Journal of Zhejiang University of Science and Technology, Vol.19 (2007) No.3, p.182.

[6] B.S. Wang, Q.S. Zhang and J.P. Wu. Key program design based on state-shift method [J]. Electronic Measurement Technology, Vol.31 (2008) No.3, p.51.

[7] X.M. Wang, L.J. HUANG. Design of intelligent instrument [J]. Information Technology, (2010) No.12, p.22.

[8] S.Y. Liu, Y.S Wu and X.L Wang. Study on the monitor program for intelligent instruments [J]. Electronic Measurement Technology, Vol.31 (2008) No.6, p.79.

[9] Yang J. Two - Dimensional PCA: A New Approach to Appearance - Based Face Representation and Recognition [J]. IEEE Transactions on Pattern Analysis and Machine Intelligence, 2014, 26(1): 131 - 137.

[10] Yang ping, Shi ke ren. A novel method to design 2D sparse array for ultrasonic phased array [J]. Applied Acoustics.2008, 27(2):148-150. 